\title{
Sistema de aquisição e processamento de imagens de ovitrampas para o combate a dengue
}

Marilú Gomes Netto Monte da Silva*, Marco Aurélio Benedetti Rodrigues, Renato Evangelista de Araujo

Resumo A dengue é uma doença viral transmitida ao homem pela picada do mosquito Aedes aegypti contaminado. A erradicação do mosquito é extremamente difícil. Assim a implementação e o acompanhamento das medidas de controle da população do inseto são essenciais. O estabelecimento de métodos de monitoração do vetor da doença é uma preocupação de vários países tropicais. No estado de Pernambuco (Brasil), a ocorrência do vetor vem sendo monitorada pelo uso de ovitrampas, armadilhas especiais para a deposição e contagem dos ovos do mosquito. No entanto, comumente esta contagem é realizada manualmente com o auxílio de lupa ou microscópio, método trabalhoso que exige tempo de profissionais treinados e está sujeita a variações. Este trabalho apresenta uma ferramenta capaz de adquirir e armazenar imagens das palhetas das ovitrampas, realizar a contagem semi-automática e automática dos ovos, sem a utilização do microscópio. O sistema desenvolvido é baseado em uma plataforma óptica, uma interface homem-máquina e um software de aquisição de imagem, com a contagem assistida dos ovos do mosquito. Esta contagem semi-automática gerou um ganho de velocidade na contagem de três vezes. As informações obtidas pelo sistema são enviadas pela Internet para um computador servidor $W E B$, onde são analisadas por técnicas de processamento de imagens. A contagem automática dos ovos baseia-se nos processos de segmentação, filtragem e quantificação. Este método foi aplicado em um conjunto de 100 imagens obtendo um erro global de 2,67\%. Dois protótipos do sistema foram instalados e implementados, em duas diferentes cidades do estado de Pernambuco.

Palavras-chave Dengue, Instrumentação, Processamento de imagens, Contagem automática.

\section{System for acquisition and processing of ovitraps images to fight dengue}

\begin{abstract}
Dengue is a viral disease transmitted to humans by the bite of the infected Aedes aegypti mosquito. The Dengue vector eradication is extremely difficult, therefore the implementation and evaluation of public policies are important issues. New methods of monitoring disease vectors are major concern in many tropical countries. In the state of Pernambuco (Brazil), the presence of the vector has been monitored by the use of ovitraps, special traps for the mosquito eggs deposition, and eggs counting methods. One drawback of the monitoring procedure is that the egg counting method has been done manually with a magnifying glass or microscope, a laborious method requiring time of trained personnel and is subject to variations. This work presents a tool capable of acquiring and storing images of the ovitraps palettes and counting eggs, semi-automatically and automatically. The developed system is based on an optical platform, a man-machine interface, and a software for mosquito eggs counting. This semi-automatic count generated a three-fold increase in the counting speed. The obtained information is sent over the Internet to a WEB server computer, where it is analyzed using image processing techniques. The automatic counting procedure is based on segmentation, filtering, and quantification processes. This method was applied to a group of 100 images giving a total error of $2.67 \%$. Two prototypes of the device have been installed and implemented in two different cities in Pernambuco state.
\end{abstract}

Keywords Dengue, Instrumentation, Image processing, Automatic counting. 


\section{Introdução}

A dengue é uma doença causada por um vírus que se aloja no mosquito Aedes aegypti e é transmitida aos seres humanos por meio da picada do inseto contaminado. O Aedes aegypti surgiu na África e de lá espalhou-se para Ásia e Américas, principalmente através do tráfego marítimo. Durante o século XVIII chegou ao Brasil juntamente com as embarcações que transportavam africanos para trabalho escravo.

O Aedes aegypti se caracteriza como um inseto de comportamento tanto urbano quanto rural. Uma vez com o vírus da dengue, a fêmea torna-se vetor permanente da doença, bem como seus descendentes, que podem nascer infectados. Os ovos do Aedes aegypti têm a peculiaridade de poderem permanecer inativos por até um ano, sem entrar em contato com a água (Perich et al., 2007). Os ovos do mosquito não são postos na água. São depositados milímetros acima de sua superfície, em recipientes como latas e garrafas vazias, pneus, calhas, caixas d'água descobertas ou pratos de vasos de plantas. Quando chove, o nível da água nesses reservatórios sobe e entra em contato com os ovos que eclodem em pouco mais de 30 minutos. Em um período de cinco a sete dias, a larva passa por quatro fases até dar origem a um novo mosquito (Dibo et al., 2005).

A erradicação do Aedes aegypti é considerada praticamente impossível, devido ao crescimento populacional e a deficiência na infra-estrutura de saneamento básico (Dallazuanna et al., 2008; Regis et al., 2008a). Contudo, o monitoramento do vetor da dengue faz-se necessário para o planejamento e a avaliação da eficácia das políticas públicas de controle da doença. O monitoramento da dengue pode basear-se na identificação de pacientes ou na determinação da presença (densidade) do vetor.
A análise estatística do crescimento da população de Aedes aegypti em Pernambuco, realizada pelo Centro de Pesquisas Aggeu Magalhães (CPqAM), baseia-se na contagem de ovos depositados em ovitrampas (Figura 1a), armadilhas especiais para coletar ovos do mosquito (Regis et al., 2008b). A ovitrampa consiste em um pequeno balde de plástico preto, com água, duas palhetas de madeira (Eucatex) e uma concentração de larvicida biológico (Bti-G) (Dallazuanna et al., 2008; Regis et al., 2008a). Cada ovitrampa pode coletar mais de 1.000 ovos, portanto elas também contribuem para a redução da população do mosquito. A cada quatro semanas, as palhetas das ovitrampas devem ser substituídas por agentes de saúde e levadas para um laboratório de contagem de ovos instalado no município da coleta.

Um grande inconveniente desta técnica é a forma da contagem dos ovos do mosquito ser realizada, um a um, através de uma lupa ou microscópio. Este processo é realizado de forma manual por um técnico treinado. Apesar de envolver profissionais especializados na área, o método de análise apresenta um erro significativo agregado à contagem. Além disto, o processo de contagem manual é lento, o que leva ao acúmulo das palhetas e consequentemente, depois de um tempo, os ovos passam a se desprender. A contaminação microbiana das palhetas também torna-se relevante com o passar do tempo, o que afeta a posterior contagem.

Neste trabalho é apresentado um sistema para auxiliar no controle da dengue, melhorando o processo de contagem dos ovos do mosquito Aedes aegypti. Aqui é apresentado o sistema (hardware/software) desenvolvido para aquisição de imagens das palhetas das ovitrampas, com interface para uma contagem semi-automática (contagem assistida pelo computador) e um algoritmo para contagem automática dos ovos, utilizando técnicas de processamento de imagem.
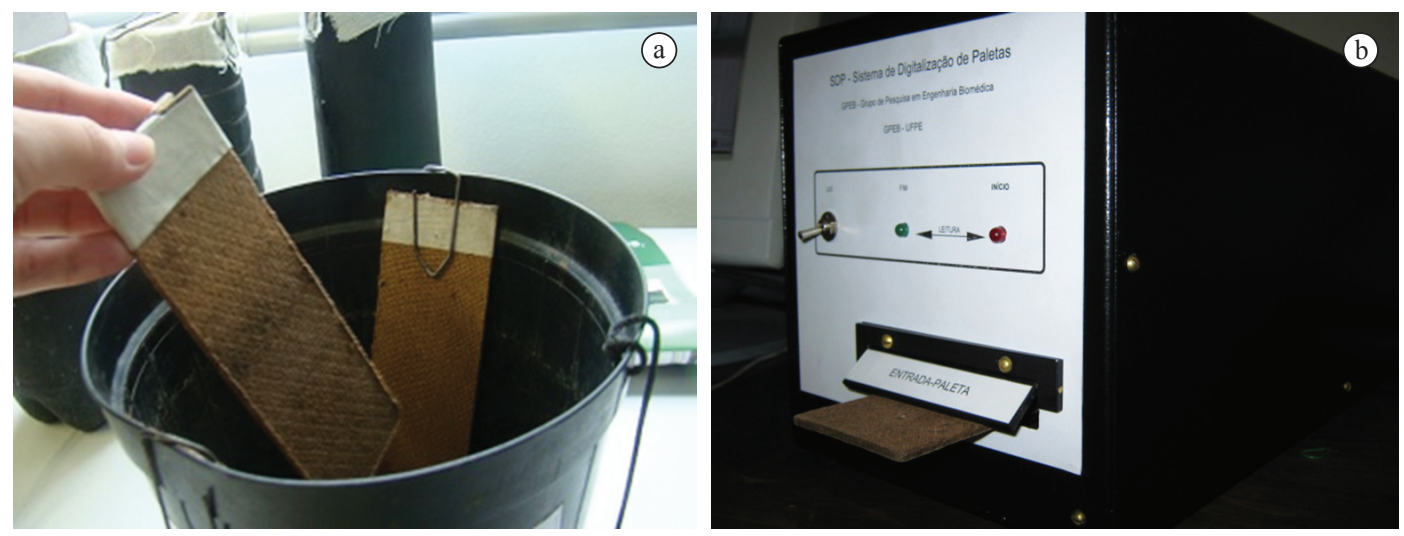

Figura 1. a) Foto de uma ovitrampa com duas palhetas, e b) painel frontal do SDP com palheta inserida no sistema. Figure 1. a) Photo of an egg trap with two blades, and b) front panel of the SDP with a palette inserted in the system. 
Existem outros algoritmos mais simples de contagem automática, baseados em sistemas de cores, que podem ser visualizados nos seguintes trabalhos: Mello et al. (2008, 2009), Portela et al. (2009) e Santos et al. (2008). Um resumo desses trabalhos, bem como informações mais detalhadas do trabalho aqui apresentado pode ser consultado em Silva (2011).

O Sistema de Digitalização de Palhetas (SDP) apresentado neste trabalho é capaz de otimizar o processo de contagem evitando o acúmulo de palhetas e apresenta de forma geo-referenciada informação sobre a evolução populacional do mosquito transmissor da dengue. O sistema desenvolvido foi implantado em duas diferentes regiões de risco da doença no estado de Pernambuco, Santa Cruz do Capibaribe e Recife. Em Recife é realizada a digitalização de palhetas e contagem de ovos de ovitrampas provenientes do município de Ipojuca (Porto de Galinhas) e Ilha de Fernando de Noronha.

\section{Materiais e Métodos}

Nesta seção é apresentado o sistema de aquisição de imagens de palhetas de Ovitrampas e descrito o algoritmo para contagem assistida e automática dos ovos da dengue.

\section{Sistema de aquisição}

O sistema para aquisição de imagens de ovos nas palhetas é apresentado no diagrama da Figura 2. O hardware de controle é composto pela placa de controle, dispositivo de captura de imagem (câmera), sistema de iluminação e mecânica de suporte para a translação de palhetas. O circuito de controle é baseado em um firmware gravado em um microcontrolador (PIC16F876), que ativa o sistema mecânico e o dispositivo de captura de imagem.

O sistema mecânico consiste em uma plataforma de translado linear motorizado, onde são colocadas as palhetas das ovitrampas. O sistema mecânico também permite um controle fino da altura da posição da câmera (controle de foco), utilizado na calibração do sistema. O hardware possui um sistema de iluminação com LEDs brancos que iluminam uniformemente a superfície da palheta.

O dispositivo de captura de imagem utilizado é composto de uma câmera digital com 7,1 megapixels e modo "super-macro" de operação. Este sistema permite a obtenção de imagens de alta resolução das palhetas, posicionadas próximas à câmera (distância de $3 \mathrm{~cm}$ ). $\mathrm{O}$ critério de escolha da câmera foi baseado em dois parâmetros: a qualidade e o tamanho da imagem. O dispositivo de captura escolhido permite a obtenção de imagens com tamanho de $3072 \times 2304$ pixels, e tamanho de arquivo da ordem de 3,5 MB, em formato JPEG. O tamanho do arquivo gerado viabiliza o custo computacional para análise automática da imagem.

O sistema foi integrado em uma caixa de metal $(30 \mathrm{~cm} \times 16 \mathrm{~cm} \times 18 \mathrm{~cm})$, permitindo ao usuário o acesso apenas à porta da plataforma, onde as palhetas são introduzidas no sistema (Figura 1b). Na parte posterior do equipamento, encontra-se um conector DB-9 para comunicação serial. Foi utilizado um cabo com conversor USB/SERIAL para realizar a comunicação com o computador via porta USB. O sistema é acionado por meio de um software, residente

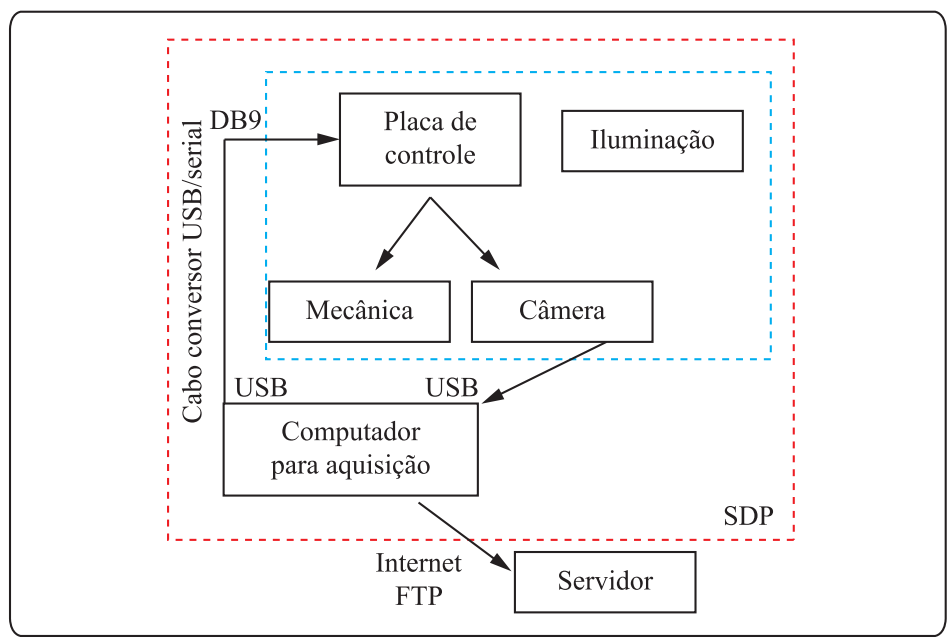

Figura 2. Diagramação do sistema de digitalização de palhetas: placa de controle, mecânica de suporte e deslocamento e câmera fotográfica digital; computador para aquisição e transferência dos dados pela internet via FTP para um servidor.

Figure 2. Diagram of the palette scanning system: control board, mechanical support and shift and digital camera; computer for acquisition and transfer of data over the Internet via FTP to a server. 
em um computador do tipo IBM-PC. O computador também recebe as imagens capturadas pelo sistema e as envia no padrão JPEG, para um sistema servidor, que por sua vez realizará a contagem automática dos ovos.

O hardware é controlado por firmware e software, tornando todo o processo automatizado. A interface homem-máquina foi desenvolvida de forma a proporcionar um controle total das funções do equipamento.

A aquisição das imagens inicia-se quando o usuário insere a palheta no sistema, o software envia a informação pela porta USB para o microcontrolador, que aciona por sua vez o sistema de controle do motor de passo, que desloca a plataforma com a amostra. A câmera fotográfica é ligada e acionada, capturando-se assim imagens da palheta. Devido ao tamanho da amostra $(12 \times 5 \mathrm{~cm})$ e a necessidade de aquisição de imagens de alta qualidade, toda a palheta é registrada com a aquisição de três imagens de regiões distintas da mesma. Após a digitalização de seções da palheta, as imagens são justapostas, por meio de sobreposição das partes comuns entre elas através de um deslocamento de uma sobre a outra, para compor uma única imagem da palheta completa. A imagem final gerada possui dimensões de $5071 \times 2304$ pixels e tamanho do arquivo de 4,5 MB. Todo o processo de captura e digitalização dura aproximadamente 40 segundos.

\section{Contagem assistida}

A interface desenvolvida permite ao usuário registrar os dados referentes à palheta, adquirir e ampliar a imagem para observar os ovos (Figura 3). Além disso, a interface permite ao usuário realizar uma contagem manual, assistida pelo computador, do número de ovos na imagem capturada. Na contagem assistida, os ovos são identificados a cada clique do mouse sobre a imagem da palheta, ficando as posições dos ovos registradas por pontos vermelhos. Na Figura 3, pode-se distinguir o que é palheta e o que é ovo.

Após a digitalização e contagem assistida pelo computador, as imagens e os dados são enviados via web por protocolo FTP (File Transfer Protocol) para um servidor na Universidade Federal de Pernambuco. Essa forma de transferência, uma das mais usadas na internet, imprime rapidez ao processo de envio dos arquivos. No servidor, as imagens são armazenadas para posterior processo de reconhecimento e contagem automática das estruturas.

Neste estudo, todas as imagens de palhetas utilizadas foram adquiridas sob as mesmas condições após terem passado um mês em campo.

\section{Contagem automática}

O processo de contagem automática estabelecido foi constituído de três etapas. A primeira etapa do algoritmo desenvolvido é a segmentação, que é

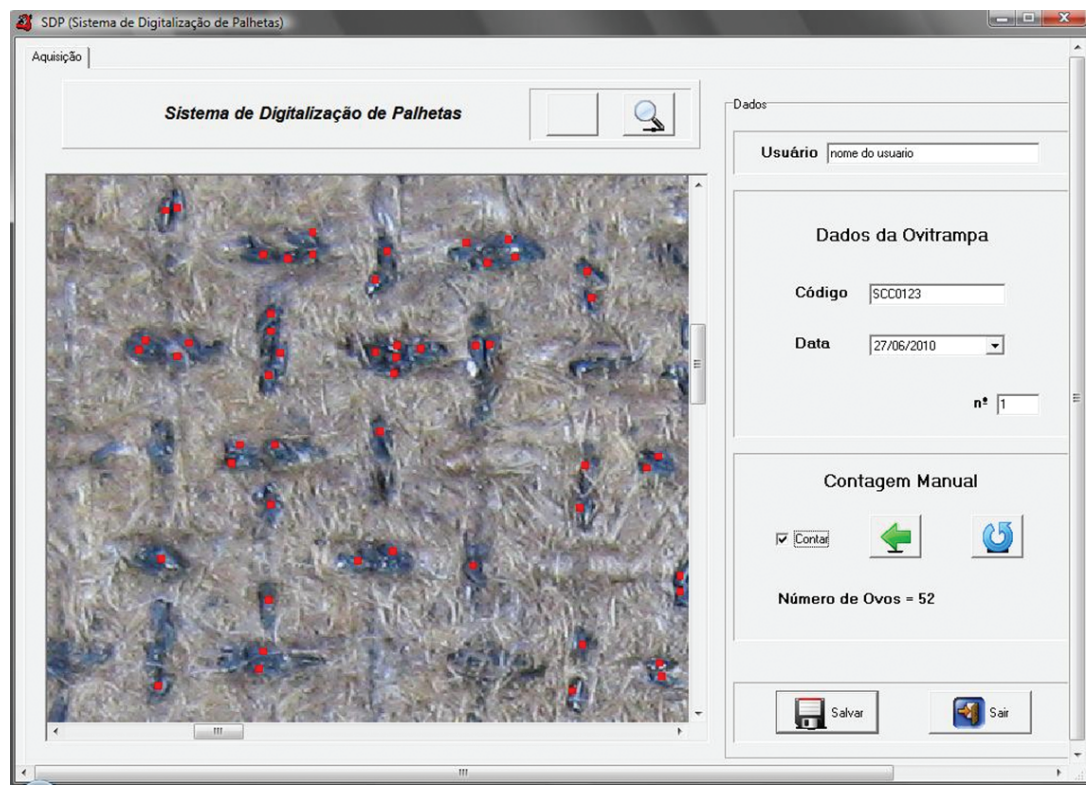

Figura 3. Interface Homem-Máquina para aquisição e contagem assistida de ovos após a aquisição de uma imagem de palheta, onde podem ser visualizados alguns ovos marcados com pontos vermelhos.

Figure 3. Human-Machine Interface for acquisition and assisted counting of eggs after the acquisition of an image vane, where some eggs marked with red dots can be seen. 
composta por uma operação AND entre duas máscaras geradas por duas formas de segmentação diferentes, a segmentação por cor e a segmentação por limiarização. Após a segmentação, é realizada uma filtragem espacial, seguida de uma filtragem morfológica. Finalmente determina-se a quantidade de ovos da imagem. Um diagrama das etapas do algoritmo pode ser observado na Figura 4.

Para a realização dos testes do algoritmo deste novo método foi utilizado um conjunto de 100 imagens. Esse conjunto representa a diversidade encontrada no acervo de imagens adquiridas no Centro de Pesquisas Ageu Magalhães (CPqAM). A seguir, são apresentadas detalhadamente as três etapas do algoritmo mencionadas.

$1^{a}$ etapa: Segmentação - Esta etapa do processamento faz uso de duas técnicas de segmentação: uma de caráter global (Gonzalez e Woods, 2008), que realiza uma pré-classificação com base no conhecimento prévio das cores dos pixels dos ovos, analisadas no espaço RGB (Gomes e Velho, 1994; Gonzalez e Woods, 2008); e outra de caráter local, que se utiliza da técnica de limiarização do histograma da imagem (Gonzalez e Woods, 2008). $\mathrm{Na}$ etapa de segmentação, assume-se que existem duas classes de estruturas na imagem: ovos (objeto) e palheta (fundo).

a) Segmentação por cor (Processamento Global) - Os histogramas das bandas R, G e B foram observados de forma separada, com a finalidade de realizar uma pré-classificação da imagem eliminando pixels referentes a níveis de cinza que não correspondessem a ovos. Após uma análise das imagens do acervo adquirido, constatou-se que as cores presentes nos ovos seguem as relações apresentadas na Equação 1 entre suas componentes RGB: $\left\{\begin{array}{l}r(i, j)<0,8 b(i, j) \\ g(i, j)<0,79 b(i, j)\end{array}\right.$

com $r(i, j), g(i, j)$ e $b(i, j)$ sendo as componentes de cor vermelha, verde e azul, respectivamente, do pixel localizado na posição $(i, j)$. A aplicação da condição apresentada na Equação 1 resulta em uma máscara que identifica possíveis pixels provenientes de ovos. Com isto, os pixels improváveis de serem ovos são rejeitados.

b) Segmentação por limiarização (Processamento Local) - Neste passo, a imagem total da palheta é subdividida em janelas e a análise ocorre em cada subdivisão. O tamanho da janela não pode ser muito pequeno, pois as características "globais" das classes desaparecem. Por outro lado, elas também não podem ser muito grandes, pois as características locais de setores da imagem tornam-se indistinguíveis, impossibilitando um tratamento diferenciado para cada setor. Experimentalmente, um bom tamanho encontrado foi $100 \times 100$ pixels.

Através da observação dos histogramas de diversas imagens do acervo, constatou-se que a distribuição das intensidades das componentes de cor vermelha dos pixels da palheta assemelha-se a uma Gaussiana (Soong, 2004), Figura 5. A imagem correspondente a essa componente detém maior contraste entre o objeto e o fundo, fato este determinante para uma maior eficiência do algoritmo de segmentação.

A caracterização da Gaussiana (histograma) é estabelecida com a determinação da sua média (que coincide com a moda) e do seu desvio padrão. Os valores de média $\left(\mu_{G}\right)$ e desvio da gaussiana $\left(\sigma_{G}\right)$ são determinados pelas Equações 2, respectivamente.

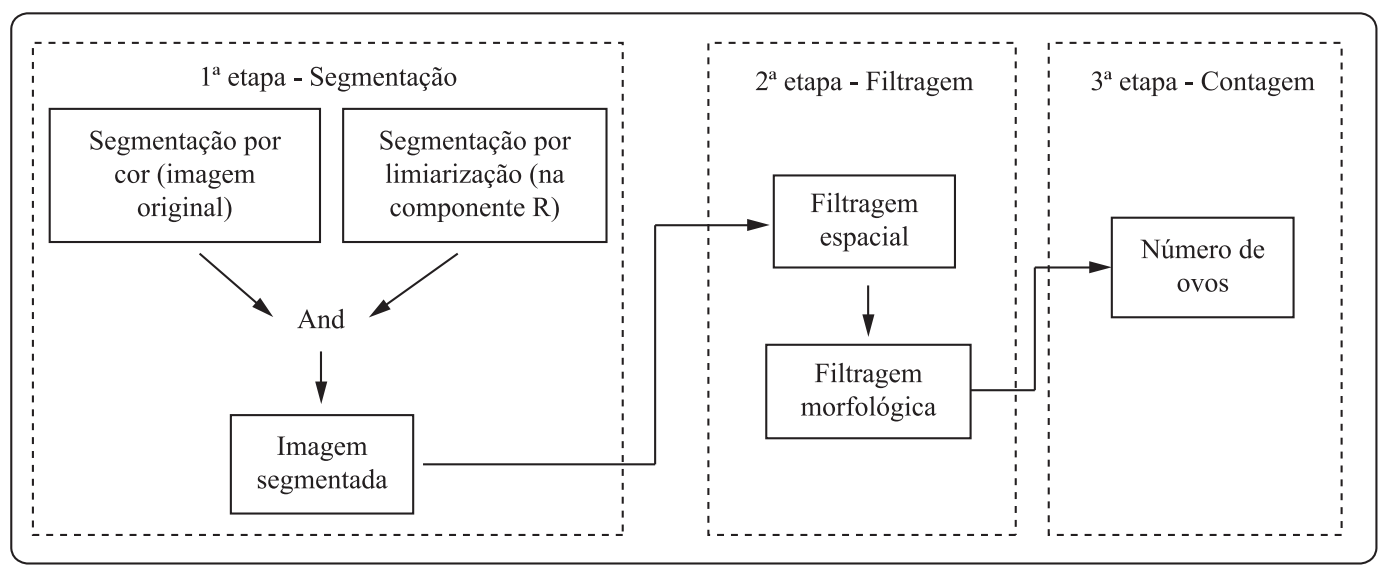

Figura 4. Diagrama das etapas do algoritmo de Contagem Automática. Figure 4. Diagram of the stages of the Automatic Counting algorithm. 


$$
\begin{aligned}
\mu_{G} & =\sum_{i=\operatorname{moda}-5}^{\bmod a+5} \overline{i p_{i}} \\
\sigma_{G} & =\sqrt{\sum_{i=\mu_{G}}^{255}\left(i-\mu_{G}\right)^{2} \hat{p}_{i}}
\end{aligned}
$$

$$
\text { com, } \overline{p_{i}}=\frac{N_{i}}{\sum_{j=\bmod a-5}^{\operatorname{moda}+5} n_{j}} \text { e } \hat{p}_{i}=\frac{n_{i}}{\sum_{j=\mu_{G}}^{255} n_{j}}
$$

sendo $n_{i}$ o número de pixels com intensidade de cor " $i$ " presentes na janela em análise.

Nesse processo de limiarização, constatou-se que os pixels dos ovos, em sua maioria, têm cores escuras, ou seja, com baixa intensidade. Desta forma, em uma imagem de palheta com ovos, a distribuição de intensidades correspondente aos ovos se sobrepõe ao lado esquerdo da distribuição Gaussiana relacionada à palheta, como mostrado na Figura 6 . $\mathrm{O}$ lado direito dessa distribuição de intensidade referente à palheta (região de maior intensidade) fica praticamente inalterado pela presença dos ovos na imagem. Como a Gaussiana do histograma de intensidade da palheta é simétrica, pode-se utilizar o lado "intacto" para a determinação da média e do desvio do histograma referente à região da palheta (sem ovos), para a determinação de $\mu_{G}$, de $\sigma_{G}$ e do limiar do processo de segmentação.

Ainda, complementando a análise, percebe-se que se a janela contivesse apenas pixels da palheta, a distribuição teria uma dada entropia (Soong, 2004). Com o aparecimento de ovos na imagem, a entropia se eleva, pois a distribuição se torna mais dispersa.
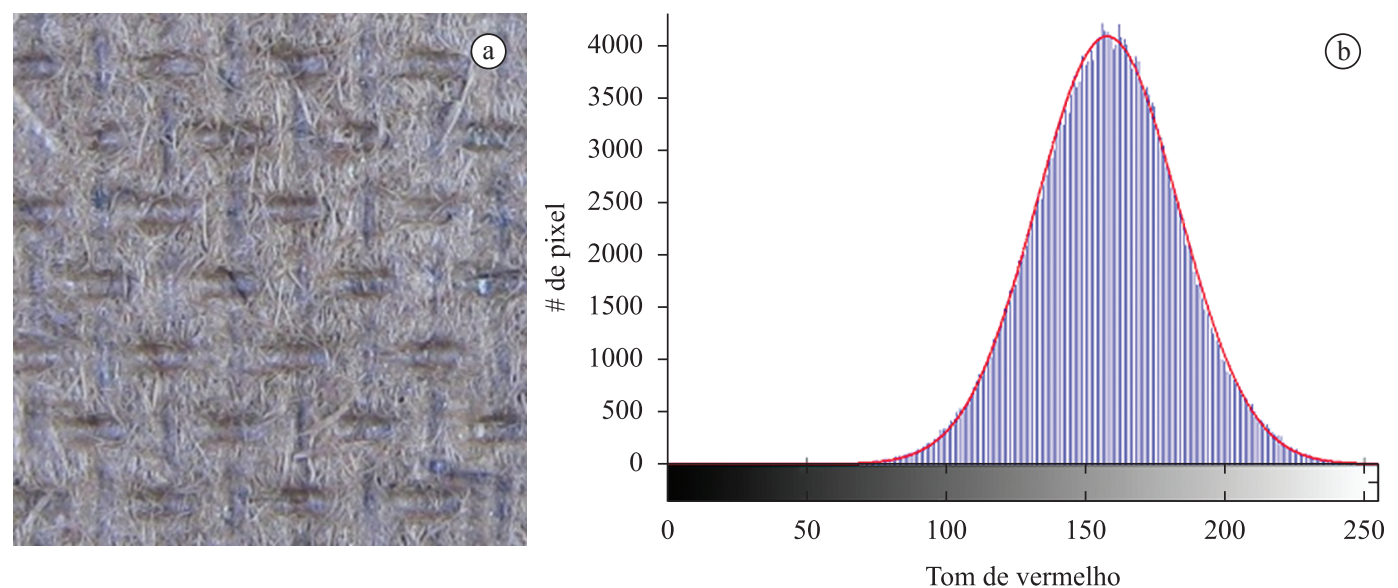

Figura 5. a) Imagem da palheta sem ovos e b) seu histograma da componente vermelha. Figure 5. a) Image of the reed without eggs and b) its histogram of the red component.

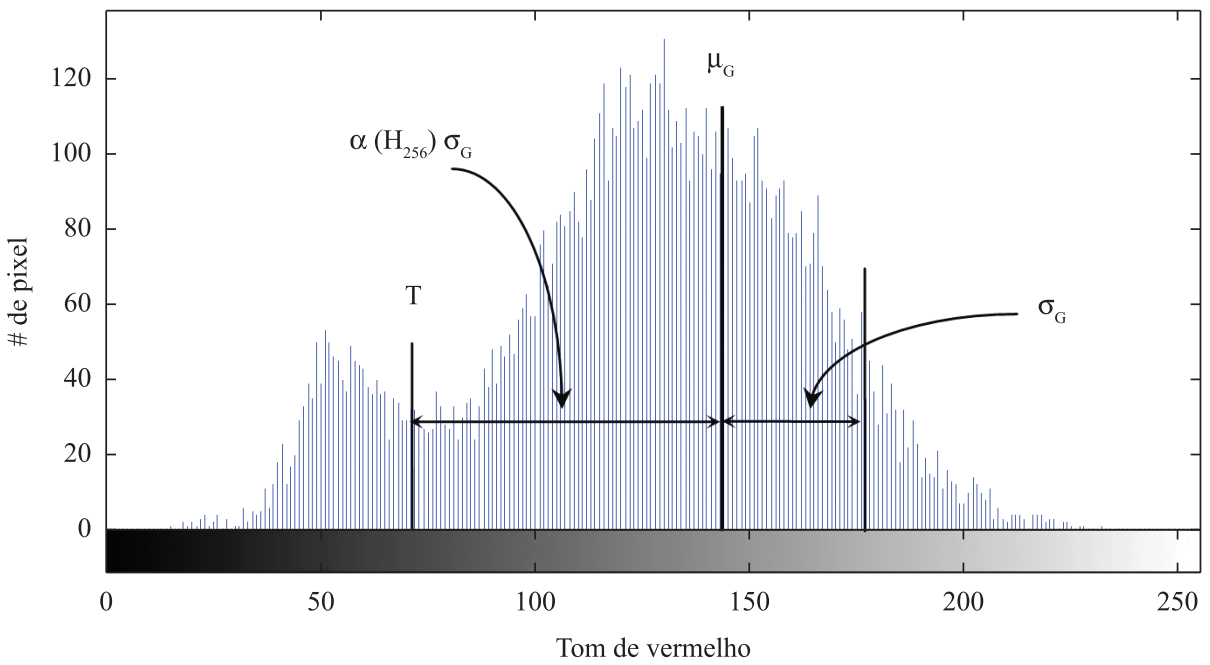

Figura 6. Histograma de uma janela $100 \times 100$ pixels da imagem da palheta com ovos. Figure 6. Histogram of a $100 \times 100$ pixels image of a palette with eggs. 
Como o algoritmo é baseado em limiarização, em cada janela da imagem, levanta-se seu histograma e determina-se um limiar. Esse limiar é uma intensidade de cor limite que determina a seguinte condição: se a intensidade do pixel em análise é superior à do limiar, ele é classificado como palheta, caso contrário ele é classificado como ovo. Para a determinação desse limiar, foi utilizado o modelo de distribuição aqui apresentado, baseado nas distribuições dos pixels da palheta e da janela como um todo.

O limiar de intensidade utilizado para distinguir ovos e palheta no histograma de intensidade é determinado pela Equação 4, que estabelece que o limiar $(T)$ é a média da gaussiana $\left(\mu_{G}\right)$ subtraída de um fator $(\alpha)$ vezes o seu desvio $\left(\sigma_{G}\right)$.

$T=\mu_{G}-\alpha\left(H_{256}\right) \sigma_{G}$

Deve-se ressaltar que, como a análise é feita janela a janela, há um limiar diferente para cada janela.

Com a presença dos ovos a entropia da distribuição aumenta. Ao mesmo tempo, para que as intensidades dos pixels dos ovos (que são escuros) fiquem abaixo do limiar, torna-se necessário que este limiar seja mais elevado, ou seja, que o fator $\alpha$ seja menor (vide Equação 4). Desta forma, pode-se estabelecer uma relação, exposta na Equação 5, do fator $\alpha$ com a função entropia $\left(H_{256}\right)$ (Abramson, 1963; Shannon, 1948) com seu valor normalizado, entre 0 e 1.

$\alpha\left(H_{256}\right)=\frac{1,6}{H_{256}}$

Sendo a entropia de Shannon $\left(H_{256}\right)$ expressa por:

$H_{256}=-\sum_{i=0}^{255} p_{i} \log _{256}\left(p_{i}\right)$ com $p_{i}$ sendo a probabilidade de um pixel ter a intensidade de cor $i$, expressa por

$$
P_{i}=\frac{n_{i}}{\sum_{i=0}^{255} n_{i}}
$$

e $n_{i}$ sendo o número de pixel com intensidade de cor " $i$ " presentes na janela em análise.

Destaca-se que o fator $\alpha$ foi determinado empiricamente pela análise de diversos experimentos, contabilizando-se os dados de cada uma das janelas das imagens analisadas. Supondo que $\alpha$ segue a seguinte relação:

$\alpha=K H^{n}$

E aplicando o logaritmo na Equação 8, tem-se:

$\log \alpha=\log K+n \log H$

Para determinar $K$ e $n$ foram realizados testes com cinco imagens, janela a janela, medindo os valores da média $\left(\mu_{G}\right)$, desvio da Gaussiana $\left(\sigma_{G}\right)$ e entropia $\left(H_{256}\right)$. Ainda foram determinados manualmente um limiar (T) para cada janela, a partir da visualização das imagens segmentadas, otimizando a classificação. $\mathrm{Na}$ detenção desses dados, foram calculados os valores de $\alpha$ através da Equação 4. A relação entre os valores do logaritmo de $\alpha$ e de $H_{256}$, de cada janela, é apresentada na Figura 7.

Pelo gráfico da Figura 7 obtém-se que $n \cong-1 \mathrm{e} K \cong 1,6$. Logo, a relação entre o fator $\alpha$ e a entropia $H_{256}$ é dada pela Equação 5.

$2^{a}$ etapa: Filtragem - Pelo fato de haver uma sobreposição das distribuições dos ovos e da palheta no histograma de intensidades, na etapa de limiarização,

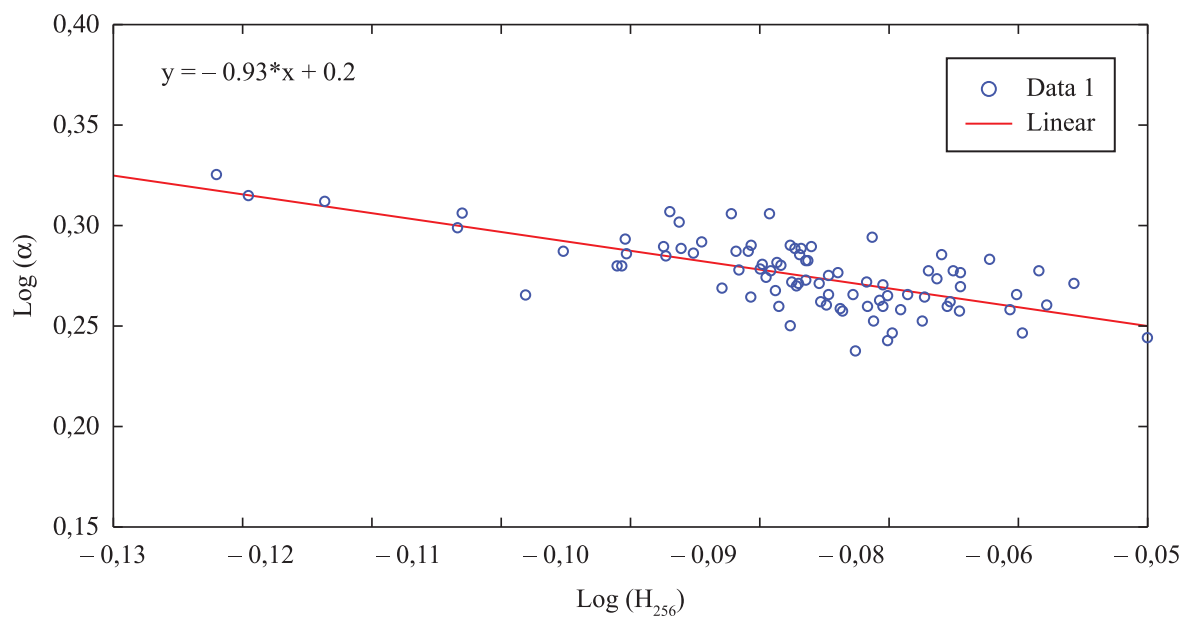

Figura 7. Gráfico do $\log \left(\mathrm{H}_{256}\right) \times \log (\alpha)$ para determinar a relação de $\alpha$ com a entropia.

Figure 7. Graph of $\log \left(H_{250}{ }^{256} \times \log (\alpha)\right.$ to determine the relationship between $\alpha$ and the entropy. 
alguns pixels podem ser classificados de forma equivocada. Geralmente esses pixels se apresentam de forma isolada ou em pequenos grupos. Esta segunda etapa do processo de identificação e contagem de ovos visa eliminar falhas do processo de limiarização.

a) Filtragem espacial (Filtro de moda) - Para eliminar esse ruído, ou seja, pixels do objeto que foram classificados como fundo e vice-versa (etapa 1), realizou-se uma filtragem com o filtro de moda de dimensões $3 \times 3$ pixels (Gonzalez e Woods, 2008). Esse tamanho de janela foi o que proporcionou melhores resultados na identificação dos ovos.

b) Filtragem morfológica (Abertura) - Foi utilizado um filtro baseado em morfologia matemática, operação de abertura (Gonzalez e Woods, 2008), com a finalidade de suavizar o contorno dos objetos, separar regiões conectadas e eliminar pixels ou grupo de pixels que ainda permaneceram após a primeira filtragem. Nesta operação, foi utilizado um elemento estruturante no formato de disco com raio de 2 pixels.

$3^{a}$ etapa: Contagem dos ovos - Esta etapa é referente à contagem dos ovos do mosquito, após os processos de segmentação e filtragens. Nesta etapa, a quantidade de pixels pertencentes aos ovos é dividida pela quantidade de pixels média de apenas um ovo, conforme Equação 10.

$$
N_{\text {ovos }}=\frac{A_{\text {totalovos }}}{A_{\text {ovo }}}
$$

sendo $N_{\text {ovos }}$ o número total de ovos na imagem, $A_{\text {total ovos }}$ $\mathrm{a}$ área (em pixels) ocupada pelos ovos da imagem $\mathrm{e}$ $A_{\text {ovo }}$ a área média (em pixels) ocupada por um ovo. Foi necessária uma avaliação prévia das imagens para a determinação da área média de um ovo. Tal área foi determinada através da síntese de imagens contendo apenas ovos, gerada manualmente no software Corel $^{\circledR}$ PHOTO-PAINT X3 (Corel Corp). Para essa síntese de imagem, foram utilizadas 100 imagens de ovos e determinou-se um valor médio de 160 pixels por ovo. Este valor médio pode variar ao se tratar de imagens com diferentes resoluções.

\section{Resultados e Discussão}

Para a validação dos resultados, dois dos três protótipos do sistema desenvolvido foram implantados em duas diferentes áreas de risco da dengue.

No CPqAM, foram adquiridas aproximadamente 6600 imagens de palhetas com o sistema SDP por profissionais experientes no reconhecimento de ovos do mosquito da dengue. Os ovos foram contabilizados com o Sistema de contagem assistida e enviados através da internet para o servidor, que realiza a aplicação dos algoritmos de contagem automática.
Para verificar a eficiência do sistema foi realizada uma comparação entre os tempos necessários para contagem manual dos ovos com o Sistema de contagem assistida e o método convencional (lupa ou microscópio). Com a utilização no $\mathrm{CPqAM}$, pode se ter, em média, o tempo que um técnico leva para realizar a contagem com o sistema de contagem semi-automática, cerca de 9,6 minutos por palheta. A contagem realizada com uma lupa ou microscópio leva em média 26 minutos, considerando apenas as contagens sem interrupção.

Verificou-se maior velocidade, no mínimo três vezes mais rápida, na contagem do número de ovos em palhetas com o sistema desenvolvido, ainda sendo melhorado de acordo com a prática do usuário em utilizar o sistema. Alguns fatores influenciam nesse tempo de contagem: o número de ovos, a quantidade de impurezas (sujeira) nas palhetas e a sobreposição dos ovos.

No processo de contagem automática, as etapas de segmentação (por cor e por limiarização), filtragem (espacial e morfológica) e contagem foram executadas no acervo de 100 imagens.

$\mathrm{Na}$ Figura 8 são apresentados os resultados referentes às etapas desenvolvidas no processo de contagem automática de ovos em uma parte da imagem $(512 \times 512$ pixels $)$ adquirida da palheta (Figura 8a). Os processos de segmentação são executados na componente vermelha da imagem original (Figura 8b). As Figuras 8c, d apresentam os resultados das segmentações por cor e por limiarização, respectivamente. $\mathrm{O}$ resultado final da segmentação, que é a máscara que identifica os pixels referentes aos ovos e obedece as duas condições determinadas pelas duas técnicas aplicadas, pode ser observado na Figura 8e. Esta última máscara é conseguida através de uma operação AND entre as duas máscaras, As Figuras $8 \mathrm{c}, \mathrm{d}$, obtidas anteriormente. O processo de filtragem espacial (moda), aplicado ao resultado da segmentação, é apresentado na Figura 8f. Na Figura 8g é apresentado o resultado da operação de abertura (filtragem morfológica).

Para uma melhor análise do método de processamento desenvolvido, na Figura 9 são apresentadas duas imagens representativas do acervo (RGB) e os resultados obtidos com o algoritmo automático (imagens binárias). As imagens apresentadas representam conjuntos de amostras com grande e pequena quantidade de ovos. Em particular, nas Figuras 9a, b, pode ser observada a capacidade de detecção de ovos pelo algoritmo, mesmo quando na imagem aparecem manchas de cores próximas à cor dos ovos. Nas Figuras 9a, b, são apresentadas imagens (RGB e binária) de amostra com poucos ovos. Por meio destas imagens, pode ser observada a capacidade de rejeição de elementos que não são ovos pelo algoritmo. 
O resultado da contagem automática foi comparado com os valores obtidos pelo processo de contagem manual assistida pelo computador (realizada por profissionais qualificados). Na Tabela 1, podem ser observados os resultados obtidos na contagem de ovos em 10 imagens.

$\mathrm{Na}$ análise de 100 imagens, constatou-se que o novo método automático de contagem aqui apresentado obteve um erro médio de $16,26 \%$ em relação ao resultado adquirido pelo método manual assistido pelo computador, sendo este um resultado satisfatório do ponto de vista qualitativo. Outra análise que pode ser feita é a dispersão desse erro médio através do cálculo do desvio padrão, que atingiu 12,57\%. Para a aplicação do sistema desenvolvido, faz-se necessária a avaliação do erro global (\%) da contagem automática, referente à diferença (método automático e manual) percentual na contabilização do total de ovos de todas as amostras (imagens de tamanho $512 \times 512$ pixels), referentes às duas palhetas constituintes de uma ovitrampa. Por meio deste cálculo obteve-se um erro global (erro médio de cada ovitrampa) de $2,67 \%$. Após a contabilização de todos os ovos de cada município analisado, esses dados serão enviados a um sistema georeferenciado, denominado SAUDAVEL (Regis et al., 2008a), para construção de um mapa indicando diferentes áreas de risco do vetor da dengue.
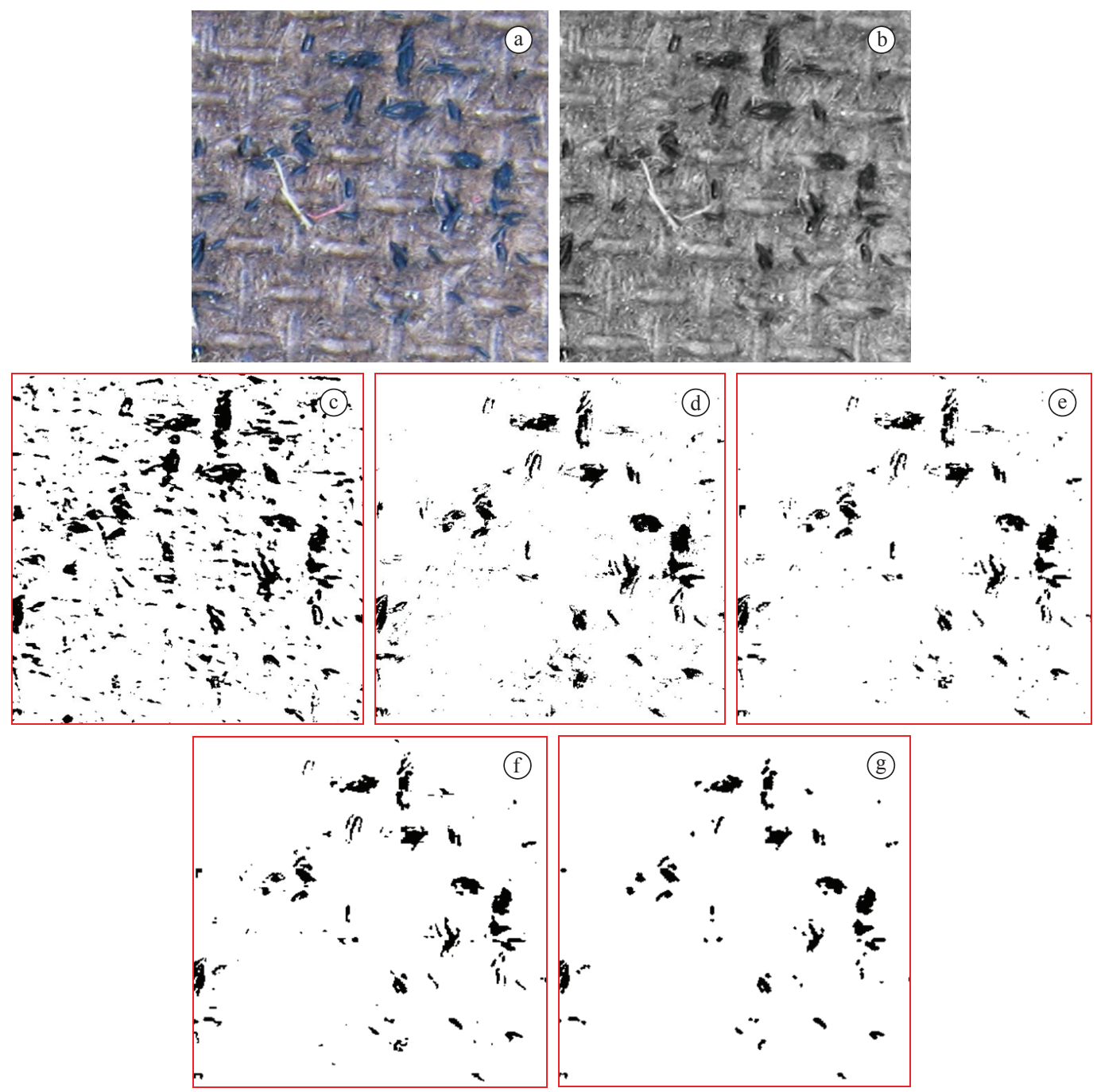

Figura 8. a) Imagem em RGB, b) componente vermelha, c) máscara resultante segmentação por cor, d) mascara resultante da segmentação por limiarização, e) resultado final da segmentação (operação AND entre (c) e (d)), f) resultado da aplicação do filtro da moda (3 × 3 ) em (e), g) resultado da filtragem morfológica em (e).

Figure 8. a) Image in $R G B$, b) red component, c) color segmentation mask, d) segmentation result by thresholding, e) segmentation final result $($ AND operation between $(c)$ and $(d)), f)$ result after applying the mode filter $(3 \times 3)$ to $(e), g)$ result of the morphological filtering applied on (e). 

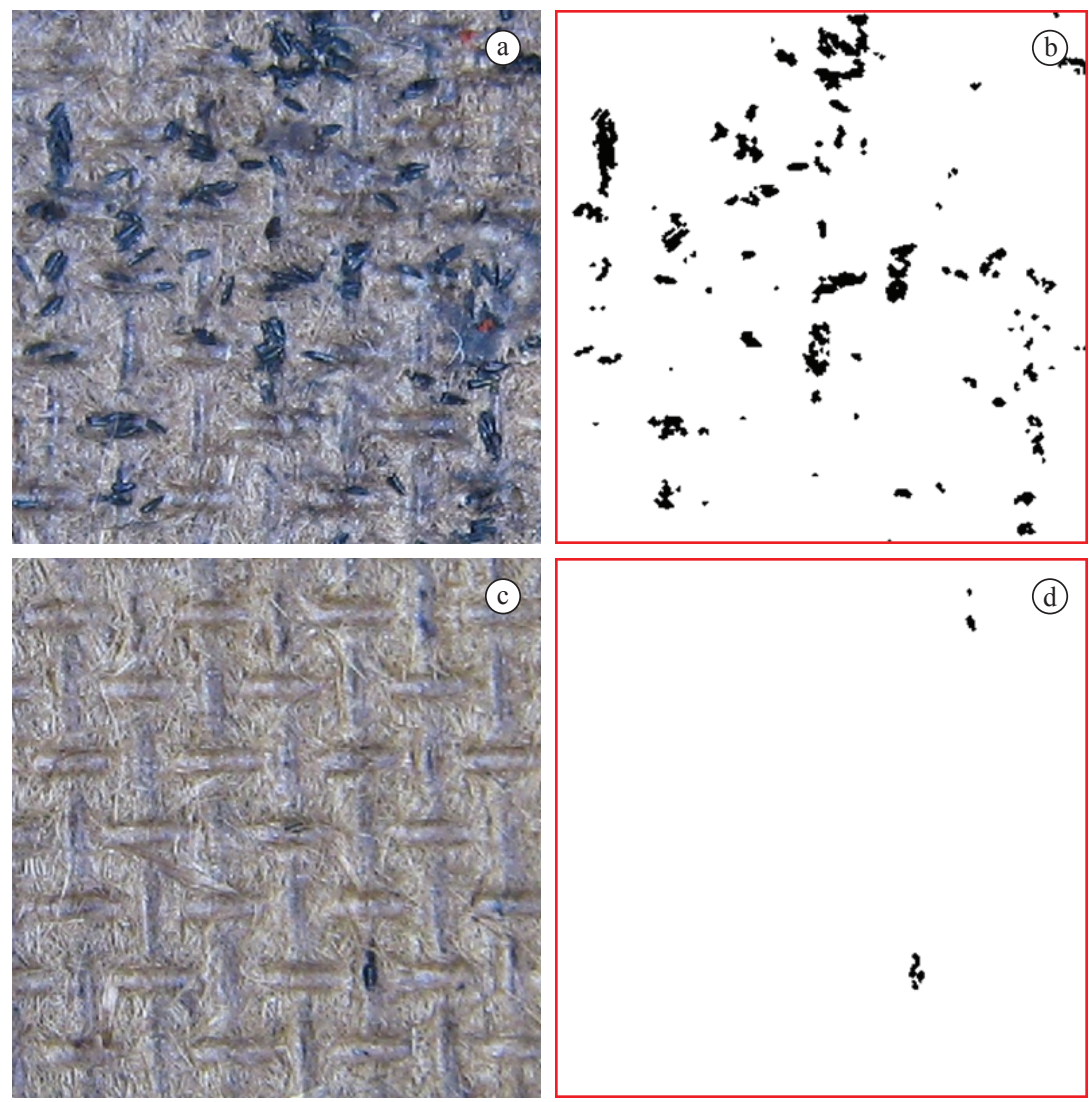

Figura 9. a) e c) Imagem original em RGB, b) e d) imagem final binária. Figure 9. a) and c) $R G B$ original image, b) and d) final binary image.

Tabela 1. Diferença quantitativa entre os métodos de contagem manual e automático.

Table 1. Quantitative difference between manual and automatic counting.

\begin{tabular}{cccc}
\hline Imagem & Manual & Automático & Diferença \\
\hline 1 & 75 & 71 & 4 \\
2 & 42 & 42 & 0 \\
3 & 30 & 33 & 3 \\
4 & 26 & 27 & 1 \\
5 & 18 & 18 & 0 \\
6 & 15 & 16 & 1 \\
7 & 9 & 8 & 1 \\
8 & 8 & 10 & 2 \\
9 & 4 & 4 & 0 \\
10 & 1 & 1 & 0 \\
\hline
\end{tabular}

\section{Conclusão}

O Aedes aegypti é um mosquito que quando infectado, é causador de uma doença que não possui tratamento específico, a dengue, tornando assim o monitoramento dos possíveis focos de extrema importância. A utilização de armadilhas especiais, ovitrampas, já é comprovadamente uma forma eficaz de monitoramento. A introdução de novas tecnologias pode aumentar o desempenho dessa monitoração.

Neste trabalho apresentou-se um sistema para digitalização da imagem de palhetas de ovitrampas, armazenamento remoto e contagem de ovos para avaliação da evolução da população do mosquito transmissor da Dengue. Os resultados obtidos foram considerados satisfatórios, segundo pesquisadores do Centro de Pesquisa Aggeu Magalhães. A grande vantagem é a capacidade de reprodutibilidade dos resultados, o que não é possível com a contagem manual.

Os resultados adquiridos pelo sistema desenvolvido serão integrados ao sistema de informação geográfica SAUDAVEL, que mostrará de forma geo-referenciada os focos do mosquito da Dengue. Desta forma, a evolução da população do mosquito em diferentes regiões será apresentada à Secretaria de Saúde do Estado, possibilitando rapidez na tomada de decisões e nas ações da administração pública para o combate à doença. 


\section{Agradecimentos}

Agradecimentos pelo suporte financeiro dado em parte pela FINEP e CNPQ ao abrigo do processo $\mathrm{n}^{\mathrm{o}}$ 01.08.0396.00. E ao Departamento de Eletrônica e Sistemas (Laboratório de Engenharia Biomédica) da Universidade Federal de Pernambuco pelo apoio tecnológico e estrutural.

\section{Referências}

Abramson N. Information theory and coding. New York: McGraw-Hill Book Company; 1963.

Corel Corp. [Internet]. [acesso em 2010 jul 28]. Disponível em: www.corel.com

Dallazuanna H, Bonat WH, Ribeiro Junior PJ. Dengue um ambiente para o monitoramento de ovos do mosquito Aedes aegypti. In: RBRAS: Anais da 53 ${ }^{\mathrm{a}}$ Reunião Brasileira da Sociedade Internacional de Biometria; 2008 maio 14-16; Lavras. Lavras: SBCS; 2008.

Dibo MR, Chiaravalloti-Neto F, Battigaglia M, Mondini A, Favaro EA, Barbosa AAC, Glasser CM. Identification of the best ovitrap installation sites for gravid Aedes (Stegomyia) aegypti in residences in Mirassol. Memórias do Instituto Oswaldo Cruz. 2005; 100(4):339-43. PMid:16113880. http:// dx.doi.org/10.1590/S0074-02762005000400001

Gomes J, Velho L. Computação gráfica: Imagem. Rio de Janeiro: Sociedade Brasileira de Matemática; 1994.

Gonzalez RC, Woods RE. Digital image processing. 3rd ed. New Jersey: Prentice-Hall; 2008.

Mello CAB, Santos WP, Rodrigues MAB, Candeias ALB, Gusmão CMG. Image segmentation of ovitraps for automatic counting of Aedes aegypti eggs. In: EMBS 2008: Proceedings of 30th Annual International Conference IEEE Engineering in Medicine and Biology Society; 2008 Aug. 20-24; British Columbia, Canada. Piscataway: IEEE Service Center; 2008. PMid:19163363.

Mello CAB, Santos WP, Rodrigues MAB, Candeias ALB, Gusmão CMG, Portela NM. Automatic counting of Aedes aegypti eggs in ovitraps images. In: Naik GR, organizer.
Recent Advances in Biomedical Engineering. Vukovar: In-Teh; 2009. p. 211-22.

Perich MJ, Kardec A, Braga IA, Portal IF, Burge R, Zeichner BC, Brogdon WA, Wirtz RA. Field evaluation of a lethal ovitrap against dengue vectors in Brazil. Medical and Veterinary Ontomology. 2007; 17:205-10. http://dx.doi. org/10.1046/j.1365-2915.2003.00427.x

Portela NM, Mello CAB, Santos WP, Candeias ALB, Gusmão CMG, Machado SCS, Rodrigues MAB. A new algorithm for segmenting and counting Aedes Aaegypti eggs in ovitraps. In: EMBS 2009: Proceedings of 31th Annual International Conference IEEE Engineering in Medicine and Biology Society; 2009; Minneapolis. Piscataway: IEEE Service Center; 2009. p. 6714-7.

Regis LN, Santos MAVM, Furtado AF, Souza W, Silveira JRJC, Acioli RV, Carvalho MS, Monteiro AM. Monitoramento e controle de Aedes aegypti vetor de dengue - Projeto SAUDAVEL. In: SICONBIOL: Anais do $9^{\circ}$ Simpósio de Controle Biológico; 2008a; Recife. Recife: Universidade Federal de Pernambuco; 2008.

Regis L, Monteiro AM, Melo-Santos MA, Silveira Junior JC, Furtado AF, Acioli RV, Santos GM, Nakazawa M, Carvalho MS, Ribeiro Junior PJ, Souza WV. Developing new approaches for detecting ans preventing Aedes aegypti population outbreaks: bases for surveilance, alert and control system. Memórias do Instituto Oswaldo Cruz. 2008b; 103(1):50-9. http://dx.doi.org/10.1590/ S0074-02762008000100008

Santos WP, Mello CAB, Mesquita RG, Rodrigues MAB, Candeias ALB, Gusmão CMG. Um algoritmo para contagem automática de ovos do mosquito Aedes aegypti em ovitrampas para controle da dengue. In: CBEB2008: Anais do 21 Congresso Brasileiro de Engenharia Biomédica; 2008; Salvador. São Carlos: Editora Cubo; 2008.

Shannon CA. Mathematical theory of communication. Bell System Technical Journal. 1948; 27:370-423, 623-56.

Silva MGNM. Sistema de aquisição e processamento de imagens de ovitrampas [dissertação]. Recife: Universidade Federal de Pernambuco; 2011.

Soong TT. Fundamentals of Probability and Statistics for Engineers. New York: John Wiley \& Sons Ltd.; 2004.

\section{Autores \\ Marilú Gomes Netto Monte da Silva*, Marco Aurélio Benedetti Rodrigues \\ Laboratório de Engenharia Biomédica, Departamento de Eletrônica e Sistemas, Centro de Tecnologia e Geociência, Universidade Federal de Pernambuco - UFPE, Rua Acadêmico Helio Ramos, s/n, $4^{\circ}$ andar, Cidade Universitária, CEP 50740-530, Recife, PE, Brasil.}

\section{Renato Evangelista de Araujo}

Laboratório de Óptica Biomédica e Imagens, Departamento de Eletrônica e Sistemas, Centro de Tecnologia e Geociência, Universidade Federal de Pernambuco - UFPE, Recife, PE, Brasil. 